\title{
Determinanten der Passungswahrnehmung nach dem Übergang in die Sekundarstufe II
}

\section{Markus P. Neuenschwander}

Eine hohe Passungswahrnehmung zwischen dem Entwicklungsstand von Jugendlichen und ibrer Ausbildungsumwelt gilt nicht nur als Kriterium eines erfolgreichen Übergangs in die Berufslehre, sondern auch als Bedingung für Arbeitszufriedenheit und Produktivität im Betrieb. Auf der Grundlage des Forschungsprojekts FamilieSchule-Beruf (FASE B) wird (1) in der Perspektive der Kompetenzentwicklung die Passungswahrnehmung durch schulische und soziale Kompetenzen in der Primarschule vorhergesagt. (2) In der Perspektive des Arbeitsplatzes wird die Passungswahrnehmung durch betriebliche Determinanten erklärt. Die schrittweisen Regressionsanalysen der Längsschnittdaten zeigten, dass mit dem Arbeitsplatzansatz mehr Varianz aufgeklärt werden konnte als mit dem Ansatz der Kompetenzentwicklung. Die Ergebnisse legen nahe, dass hohe Konfliktlösekompetenzen von Jugendlichen zu einer günstigen Arbeitsplatzgestaltung beitragen, welche die Entwicklung der Passungswahrnehmung positiv beeinflusst.

\section{Einleitung}

Die Passung zwischen Jugendlichen und ihrer Ausbildungssituation bzw. Arbeitstätigkeit wurde in Berufswahl- und Transitionstheorien immer wieder als Kriterium eines erfolgreichen Übergangs in die Berufsbildung und das Erwerbsleben vorgeschlagen (Holland, 1973; Super, 1992). Die Herstellung einer Mensch-Umwelt-Passung gibt der menschlichen Entwicklung eine Richtung (z.B. bei Piaget, 1947). Menschen wählen Kontexte, die mit ihren Fähigkeiten und Interessen korrespondieren, oder sie versuchen, diese Kontexte nach den eigenen Zielen und Bedürfnissen zu modifizieren (Überblick in Brandtstädter, 1999). Eine hohe Passung zwischen Lernenden und ihrer Ausbildungsumwelt dürfte eine wichtige Bedingung für Produktivität in Ausbildung und Arbeit (Übersicht in Heinz, 2000), für Wohlbefinden und Ausbildungszufriedenheit (Pinquart, Juang \& Silbereisen, 2003) und für einen hohen Selbstwert (Super, 1992) sein. 
Bisher sind die entwicklungspsychologischen und kontextuellen Bedingungen einer hohen Passung in der Sekundarstufe II, namentlich in der Berufsbildung, wenig untersucht worden. Die verfügbaren Daten stammen vor allem aus US-amerikanischen Studien. Zum Schweizerischen (Berufs-) Bildungssystem fehlen dazu Informationen. Der vorliegenden Studie liegen die Fragen zu Grunde, in welchem Ausmass die schulischen und sozialen Kompetenzen von Jugendlichen eine hohe Passung zu ihrer Ausbildung bestimmen, und in welchem Ausmass diese Passung von der Gestaltung des Arbeitsplatzes im Lehrbetrieb abhängt.

In der Transitionsforschung wird Passung statisch als Korrespondenz zwischen der Persönlichkeit eines Jugendlichen und den Merkmalen eines Berufsfelds (Holland, 1973), dynamisch als Eingrenzungs- und Kompromissprozess eines Jugendlichen (Gottfredson, 1996) oder als Korrespondenz zwischen dem Entwicklungsstand eines Jugendlichen und den Ausbildungsanforderungen (Eccles et al., 1993; Eccles, 2004) definiert. Eine Passung zwischen Schüler/-in und Schule, Lernender und Ausbildung, Mitarbeitender und Betrieb ist ein zentrales Ziel der schulischen Selektion bzw. der Lehrlingsselektion. Eine Schule oder Ausbildung ist dann den Jugendlichen angemessen, wenn sie mit den altersspezifischen Bedürfnissen der aufgenommenen Jugendlichen korrespondiert (engl. stage-environment-fit). Passung beschreibt ein Mensch-Umwelt Verhältnis, das aufgrund der Veränderungen des Menschen und der Ausbildungsinstitution in ständigem Wandel begriffen ist und daher immer wieder neu hergestellt werden muss (vgl. auch Übersicht in KristofBrown, Zimmerman \& Johnson, 2005). Im Folgenden wird die Passung als Korrespondenz der Interessen bzw. Fähigkeiten eines Schülers/einer Schülerin und den Gegebenheiten der Ausbildung definiert. Passung so verstanden dürfte beim Verbleib in der gleichen Ausbildung eine gewisse Stabilität besitzen, weil sich Institutionen nur langsam verändern. Während Passung eine objektive Gegebenheit bezeichnet, berücksichtigt der Begriff der Passungswahrnehmung die Subjektabhängigkeit, d.h. verschiedene Jugendliche nehmen die gleiche Passung individuell unterschiedlich wahr. Die vorliegende Studie fokussiert auf die Passungswahrnehmung, weil diese für die Entwicklung von Jugendlichen bedeutsam ist, und weil damit eine direkte Messung der Passung ermöglicht wird (Kristof-Brown et al., 2005). Aus der Definition der Passungswahrnehmung als wahrgenommene Korrespondenz der Interessen bzw. Fähigkeiten eines Schülers/einer Schülerin und den Gegebenheiten der Ausbildung folgt, dass Jugendliche, die eine hohe Passung wahrnehmen, eher produktiv arbeiten sowie motivierter und zufriedener sind. Sie können besser mit neuen Situationen umgehen (sog. Übergangskompetenzen nach Busshoff, 1998), aber auch sicherer sein, die begonnene Ausbildung abzuschliessen (Validierung der Passungswahrnehmung). Es folgt aber auch daraus, dass die Passungswahrnehmung von Jugendlichen in Brückenangeboten geringer als diejenige von Jugendlichen in zertifizierenden Ausbildungen (Mittelschule, Berufslehre) ist, weil Jugendliche nach Abschluss der Volksschule die Brückenangebote in der Regel als Verlegenheitslösung interpretieren (Ausbildungstyp-Hypothese). 


\section{Bedingungen der Passungswahrnehmung: Kompetenz- entwicklung und Arbeitsplatzgestaltung}

Zwei wichtige Perspektiven, um die wahrgenommene Passung zu erklären, bilden die Kompetenzentwicklung und die Arbeitsplatzgestaltung. Diese werden nun ausgeführt und es werden dazu Hypothesen entwickelt und empirisch überprüft.

(1) Perspektive der Kompetenzentwicklung: Jugendliche entwickeln schulische und soziale Kompetenzen, die die Anpassung an Ausbildungsanforderungen ermöglichen. So definierte Sternberg (1984) Intelligenz als Fähigkeit, sich an neue kognitive und soziale Anforderungen anzupassen. Kognitive und soziale Fähigkeiten Jugendlicher am Ende der Primarschule, d.h. vor dem Übergang in die Sekundarstufe I, bilden Voraussetzungen, um in der Sekundarstufe II eine hohe Mensch-Umwelt-Passungswahrnehmung zu entwickeln.

(a) Schulische Kompetenzen vergrössern das Spektrum von Ausbildungsmöglichkeiten, insofern gute Schulabschlüsse den Zugang zu anspruchsvollen Ausbildungsgängen ermöglichen (Herzog, Neuenschwander \& Wannack, 2006). Konkret beeinflussen Noten das fachbezogene Fähigkeitsselbstkonzept (Helmke \& Weinert, 1997; Neuenschwander, 2005), auf dessen Grundlage sich Jugendliche für unterschiedlich anspruchsvolle Berufslehren entscheiden. Jugendliche mit einem hohen Fähigkeitsselbstkonzept trauen sich eher zu, auch hohe Leistungsanforderungen einer Berufslehre zu erfüllen und sie erleben daher eher eine hohe Passung.

(b) Soziale Kompetenzen sollen gemäss den Zielen vieler kantonaler Lehrpläne in der Schule gefördert werden. Geringe soziale Kompetenzen führen zu Unterrichtsstörungen, die in schulischen Selektionsverfahren implizite, aber bedeutsame Selektionskriterien bilden (Neuenschwander \& Malti, 2009). Überdies sind soziale Kompetenzen wie Konfliktlösungsfähigkeit wichtige Kriterien bei der Lehrstellenvergabe (Neuenschwander \& Wismer, 2010). Jugendliche mit hohen Konfliktlösefähigkeiten kennen Strategien, soziale Konflikte zu lösen, ohne frustriert und aggressiv zu werden (Alsaker, 2003). Es wird daher vermutet, dass geringe Konfliktlösefähigkeiten zu aggressivem Verhalten führen. Eine hohe Aggressionsneigung erschwert Jugendlichen nach dem Übergang in die Sekundarstufe II die soziale Integration in eine Gruppe, so dass sie eine geringere Passung zwischen sich und dem Ausbildungskontext wahrnehmen.

Die Passungswahrnehmung könnte durch askriptive Merkmale von Jugendlichen (Geschlecht, Staatsangehörigkeit) beeinflusst werden, weil Schüler und Schülerinnen bzw. Einheimische und Migranten unterschiedliche Bedürfnisse an die Ausbildung haben und Interessen verfolgen (Labudde, Herzog, Neuenschwander, Violi \& Gerber, 2000). Das Arbeitsmodell zur Veränderung der Passungswahrnehmung schliesst daher zu Kontrollzwecken das Geschlecht und die Staatsangehörigkeit von Jugendlichen ein. 
(2) Arbeitsplatzbezogene Perspektive: Die zweite Position stellt den Ausbildungskontext bzw. den Arbeitsplatz als Determinante der Passungswahrnehmung ins Zentrum. Passung ist nicht nur ein Ergebnis der individuellen Anpassungsleistung eines Jugendlichen, sondern auch der Institution. Mittelschulen, Brückenangebote und die duale Berufsbildung sind sehr unterschiedliche Ausbildungskontexte. Aus Platzgründen beschränkt sich die vorliegende Arbeit auf die duale Berufsbildung, den häufigsten Ausbildungskontext in der Schweizer Sekundarstufe II.

Das Berufsprestige bezeichnet das öffentliche Ansehen eines Berufs und bildet nach Gottfredson (1996) ein Auswahlkriterium für eine Berufslehre. Es ist aber ungeklärt, ob Jugendliche in Lehren mit hohem Berufsprestige eine höhere Passungswahrnehmung berichten. Den Studien von Eccles (2004) zur Sekundarstufe folgend, beeinflusst die Schulwahrnehmung die Mensch-Umwelt-Passung. Wenn Lehrbetriebe abwechslungsreiche Arbeitsplätze schaffen, Mitsprache gewähren und persönliche Unterstützung anbieten, aber auch Belastungen gering halten, kann eine hohe Passung entstehen. Ausserdem dürfte eine hohe Zufriedenheit des Jugendlichen mit dem Berufsbildner zu einer hohen Passungswahrnehmung beitragen, weil der Berufsbildner im betrieblichen Alltag eine wichtige Rolle spielt. Es wird also postuliert, dass Arbeitsplatzmerkmale die Passungswahrnehmung unabhängig von den schulischen und sozialen Kompetenzen von Lernenden beeinflussen. Um allfällige Effekte des Geschlechts und der Staatsangehörigkeit auszuschliessen, sollen sie analog zum ersten Ansatz auch bei der Hypothesenprüfung berücksichtigt werden.

Die Passungswahrnehmung ist also einerseits ein Erfolgskriterium der Berufswahl, andererseits ein Indikator für eine qualitativ gute Ausbildung. Zusammenfassend werden folgende Hypothesen überprüft:

1. Eine hohe wahrgenommene Mensch-Umwelt Passung in der Sekundarstufe II korrespondiert mit hohen Übergangskompetenzen, aber auch mit Wohlbefinden und Arbeitszufriedenheit sowie mit der Überzeugung, die angefangene Ausbildung abzuschliessen (Validierungshypothese).

2. Jugendliche in Zwischenlösungen (Brückenangeboten) erleben eine geringere Passung als Jugendliche in zertifizierenden Ausbildungen (Ausbildungstyphypothese).

3. Eine hohe wahrgenommene Mensch-Umwelt-Passung in der Sekundarstufe II stellt sich ein, wenn Jugendliche in der Sekundarstufe I ein günstiges Fähigkeitsselbstkonzept und geringe aggressiven Tendenzen aufweisen und in der Primarschule hohe schulische Kompetenzen und hohe Konfliktlösungskompetenzen haben (Perspektive der Kompetenzentwicklung).

4. Eine hohe wahrgenommene Mensch-Umwelt-Passung in der Berufslehre verändert sich nach Kontrolle von Noten und Konfliktlösungskompetenzen abhängig vom Berufsprestige, von der Arbeitsplatzgestaltung und von der Zufriedenheit mit dem Berufsbildner/der Berufsbildnerin (Perspektive des Arbeitsplatzes). 


\section{Methode}

Die Hypothesenprüfung erfolgte mit Längsschnittdaten des Forschungsprojekts Familie-Schule-Beruf (FASE B) ${ }^{1}$. Alle Messinstrumente sind ausführlich in Neuenschwander et al. (2003, 2007a, 2007b, 2009) beschrieben.

\section{St ichprobe}

Es wurde eine geschichtete Zufallsstichprobe verwendet, welche für die Schülerinnen und Schüler des deutschsprachigen Kantons Bern der 6. Klassenstufe repräsentativ ist. Dabei wurden im ersten Schritt 26 Schulstandorte aus den vier deutschsprachigen Inspektoraten (entspricht geografischen Regionen) und in einem zweiten Schritt in jeder Schule zwei bis drei Klassen des Zielschuljahres zufällig ausgewählt. Es nahmen insgesamt 234 Schülerinnen und 220 Schüler aus 25 Klassen teil (Durchschnittsalter 11.9 Jahre). Die erste Befragungswelle fand im Spätherbst 2002 statt.

Längsschnittstichprobe: Die gleichen Jugendlichen wurden im Frühsommer 2006 (Ende 9. Schuljahr) ein zweites Mal schriftlich befragt $(N=345)$, im Winter 2007 (1. Jahr nach Schulaustritt) im Rahmen einer Internetbefragung ein drittes $\mathrm{Mal}(N=241)$ und im Frühjahr 2008 per Post ein viertes Mal $(N=219)$. Es lagen keine systematischen Rücklaufverzerrungen zwischen den zum zweiten Messzeitpunkt teilnehmenden und den nicht teilnehmenden Jugendlichen in Bezug auf zentrale Variablen zum ersten Messzeitpunkt vor (Leistungen in Mathematik Effektstärke $\mathrm{d}=.10$, Leistungen in Deutsch $\mathrm{d}=.13$, Bildungserwartungen von Eltern $\mathrm{d}=.03$, ISEI ${ }^{2} \mathrm{~d}=.07$, Verhaltensprobleme im Unterricht $\mathrm{d}=.07$; Neuenschwander et al. 2007b). Auch von der zweiten zur dritten Erhebungswelle fanden wir keine systematischen Rücklaufverzerrungen. Von der dritten zur vierten Erhebungswelle fanden wir ebenfalls keine Rücklaufverzerrungen mit der Ausnahme, dass die Teilnahmebereitschaft von Schweizer Jugendlichen in der Erhebung 2008 höher als von ausländischen Jugendlichen war. Insgesamt nahmen 219 Jugendliche an den Schülerbefragungen aller vier Erhebungen teil. Eine detaillierte Beschreibung des Rücklaufs bzw. allfälliger Rücklaufverzerrungen über die verschiedenen Erhebungswellen findet sich in Neuenschwander und Frank (2009). Für die Überprüfung der arbeitsplatzbezogenen Hypothese wurden aus dieser Stichprobe diejenigen 126 Jugendlichen ausgewählt, die zum vierten Messzeitpunkt in einer Berufslehre waren.

\section{Instrumente}

$\mathrm{Zu}$ verschiedenen Messzeitpunkten kamen standardisierte Fragebogen zum Einsatz. Die Items konnten in der Regel auf einer vorgegebenen Ratingskala beantwortet werden. Wenige Konzepte wurden mit Einzelitems operationalisiert, insbesondere wenn konkrete Fakten abgefragt wurden (z.B. Geschlecht, Zeugnisnoten, Nationalität usw.). 


\section{Erster Messzeitpunkt}

Die Schülerinnen und Schüler gaben für die Fächer Deutsch, Mathematik, Natur-Mensch-Mitwelt (NMM), Zeichnen, Musik, Sport die letzten Zeugnisnoten an. Sie hatten als Grundlage dafür das Schulzeugnis des vorangegangenen fünften Schuljahres im Befragungszimmer verfügbar. Die Noten wurden auf einer Skala mit den Punkten 6 (sehr gut), 5 (gut), 4 (genügend), 3 (ungenügend), 2 (sehr ungenügend) und 1 (äußerst ungenügend) erfasst.

Die Sozialkompetenz wurde anhand von 21 Items von den Jugendlichen selber beurteilt. In einer Hauptachsenanalyse wurden drei Faktoren gefunden (Varianzaufklärung 27\%). Der Faktor Konfliktlösefähigkeit wurde von den Jugendlichen anhand von acht Items wie zum Beispiel «Wenn ich eine Meinungsverschiedenheit mit jemandem habe, kann ich sehr gut akzeptieren, dass der andere nicht die gleiche Meinung hat wie ich» erfasst. Es wurde eine Ratingskala mit den Polen 1: «stimmt überhaupt nicht» und 4: «stimmt genau» verwendet (Alpha=.74, M=2.98, SD=.44).

\section{Zweiter Messzeitpunkt}

In der Regel wurde eine sechsstufige Ratingskala verwendet, die zu den Polen 1 (stimmt überhaupt nicht) und 4 (stimmt voll und ganz) rekodiert wurde, um die Vergleichbarkeit mit den Daten der Erhebung zum ersten Messzeitpunkt zu vereinfachen.

Die wahrgenommene Passung mit der Ausbildung wurde mit drei Items erfasst nämlich «die Anschlusslösung ist für mich im Moment die beste Lösung», "die Anschlusslösung stimmt mit meinen schulischen Fähigkeiten überein» und «die Anschlusslösung stimmt mit meinen persönlichen Interessen überein» (Varianzaufklärung der Hauptachsenanalyse: 53\%; Alpha=.77, M=3.26, SD=.51).

Das Fähigkeitsselbstkonzept Deutsch nach Buff (1991) setzte sich aus sieben Items zusammen wie "Ich kann die Aufgaben gut lösen, die ich im Deutschunterricht bearbeiten muss» (Alpha $=.86, \mathrm{M}=3.00, \mathrm{SD}=.46)$. Die Hauptachsenanalyse ergab eine Varianzaufklärung von $49 \%$.

Das Fähigkeitsselbstkonzept Mathematik nach Buff (1991) wurde analog zum Fähigkeitsselbstkonzept Deutsch konstruiert und setzte sich aus sieben Items wie zum Beispiel «Ich bin in Mathematik gut» zusammen (Alpha=.62, M=2.80, $\mathrm{SD}=.61$ ). Die Hauptachsenanalyse ergab eine Varianzaufklärung von 62\%.

Aggressives Verhalten: Anhand von fünf Items wurden Störungen der sozialen Ordnung erfragt (Neuenschwander et al., 2003, 2007b). Die Faktorenanalyse ergab zwei Faktoren (Varianzaufklärung 56\%). Der Faktor aggressives Verhalten setzte sich aus zwei Items zusammen wie zum Beispiel «mitgeholfen, andere Jugendliche zu ärgern» (Alpha=.60, M=1.69, SD=.83). Die fünfstufige Antwortskala umfasst die Punkte 1: nie, 2: 1- bis 2-mal, 3: einige Male im Jahr, 4: mindestens einmal pro Monat, 5: mindestens einmal pro Woche.

Die Staatsangehörigkeit wurde mit folgender Frage erfasst: «Welche Staatsangehörigkeiten bzw. Nationalitäten hast Du (Pässe oder Identitätskarten)?» Alle 
Nennungen wurden anhand einer Länderliste kodiert. Alle Jugendlichen mit ausländischer Staatsangehörigkeit wurden zur Gruppe Nicht-Schweiz zusammengefasst.

\section{Dritter Messzeitpunkt}

Die wahrgenommene Passung mit der Ausbildung wurde mit drei Items erfasst, wie zum Beispiel «die Lehre/die Schule ist für mich im Moment die beste Lösung» (Varianzaufklärung der Hauptachsenanalyse: 57\%; Alpha=.63, M=3.29, $\mathrm{SD}=.55)$. Es wurde eine Ratingskala mit den Polen 1: «stimmt überhaupt nicht» und 4: «stimmt genau» verwendet.

\section{Vierter Messzeitpunkt}

Wenn nicht anders vermerkt, wurde eine sechsstufige Antwortskala verwendet und zu den Polen 1 (stimmt überhaupt nicht) und 4 (stimmt voll und ganz) rekodiert.

Die wahrgenommene Passung mit Ausbildung/Arbeit wurde mit den drei gleichen Items erfasst wie zum zweiten und dritten Messzeitpunkt. Die Skala wurde aber mit einem vierten Item «die Ausbildungs-/Arbeitssituation stimmt mit meinen beruflichen Fähigkeiten überein» ergänzt, weil manche Versuchsteilnehmende in dieser Befragungswelle nicht mehr in Ausbildung waren, sondern einer beruflichen Tätigkeit nachgingen (Varianzaufklärung der Hauptachsenanalyse betrug 67\%, Alpha=.84, $M=3.39, \mathrm{SD}=.54)$. Die für die Mittelschüler/-innen und Berufslernenden relevanten Items blieben aber unverändert. Die hohe Stabilitätskorrelation zwischen dem dritten und vierten Messzeitpunkt unterstreicht die Ähnlichkeit der Konstrukte ( $\left.\mathrm{r}_{\mathrm{t} 3-\mathrm{t} 4}=.30, \mathrm{p}<.001\right)$.

Die wahrgenommene Produktivität in der Ausbildungs- bzw. Arbeitssituation wurde mit sechs Items wie zum Beispiel «In meiner momentanen schulischen/beruflichen Situation kann ich meine Stärken sehr gut verwirklichen» (Varianzaufklärung der Hauptachsenanalyse betrug 66\%, Alpha=.90, M=3.22, SD=.52).

Die Übergangskompetenzen (angeregt von Busshoff, 1998) setzten sich aus fünf Items zusammen wie zum Beispiel "Als ich die neue Ausbildung/Arbeit/Zwischenlösung angefangen habe, konnte ich mich schnell an die neuen Leistungsanforderungen gewöhnen» (Varianzaufklärung der Hauptachsenanalyse betrug 50\%, Alpha=.74, M=3.36, $\mathrm{SD}=.41$ ).

Das subjektive Woblbefinden (Grob et al., 1991) wurde mit acht Items wie zum Beispiel «Mein Leben verläuft in rechten Bahnen» gemessen (Varianzaufklärung der Hauptachsenanalyse betrug 50\%, Alpha=.86, M=3.26, SD=.39).

Die Ausbildungszufriedenheit im Lehrbetrieb wurde mit dem Item «Wenn Sie auf das letzte halbe Jahr zurückblicken, wie zufrieden sind Sie mit der aktuellen Ausbildungssituation in ihrem Lehrbetrieb?» $(\mathrm{M}=5.53, \mathrm{SD}=1.10)$. Die Antwortskala umfasste die Pole 1 (ausserordentlich unzufrieden) und 7 (ausserordentlich zufrieden). 
Die Kontrollüberzeugung wurde mit einem einzelnen Item erfasst, nämlich: "Wie sicher sind Sie, den geplanten Ausbildungsabschluss zu erreichen?» $(\mathrm{M}=3.26, \mathrm{SD}=.59)$. Die sechsstufige Antwortskala umfasste die Pole 1 (überhaupt nicht sicher) und 6 (sehr sicher), die linear zu einer vierstufigen Skala rekodiert worden ist.

Die Investitionsbereitschaft wurde mit einem einzelnen Item erfasst, nämlich "Wie gross ist der Aufwand, den Sie für den Abschluss der laufenden Ausbildung zu leisten bereit sind?» $(\mathrm{M}=4.77, \mathrm{SD}=.99)$. Die sechsstufige Antwort umfasste die Pole 1 (überhaupt nicht gross) und 6 (sehr gross), die zu einer vierstufigen Skala rekodiert worden ist.

Schultyp: Die Schülerinnen und Schüler wurden im 2. Lehrjahr bzw. 11. Schuljahr gebeten anzugeben, in welcher beruflichen Tätigkeit bzw. Ausbildungsform sie sich befanden (Optionen: Berufslehre/Attestausbildung (58\%), Berufsmaturitätsschule (7\%), Vollzeitberufsschule (2\%), Gymnasium/Fachmittelschule (17\%), Zwischenlösung (9\%). Diese Optionen wurden zu drei Typen, Berufsbildung (incl. Berufsmaturitätsschule), Gymnasium/Mittelschule und Zwischenlösung zusammengefasst.

Die Berufslehre wurde mit der folgenden offenen Frage erfasst: «Was für eine Berufslehre, Anlehre oder Grundausbildung mit Attest absolvieren Sie?» Die Antworten wurden aufgrund der Berufsdatenbank des Bundesamtes für Statistik kodiert. Zudem wurden den einzelnen Berufen Werte aufgrund der International Standard Classification of Occupations (ISCO 88) Codes zugeordnet. Treiman (1977) entwickelte die Standard Index of Occupational Prestige Skala (SIOPS) auf Grundlage des Klassifikationssystems ISCO 88. Der Prestigerang von Berufen gilt als relativ aussagekräftig und nationenunabhängig. Dieses Verfahren wurde im vorliegenden Fall auf die Berufslehren angewandt.

Die Zeugnisnoten zum Abschluss des letzten Schuljahres wurden im Schulteil mit der Frage «Welches waren ihre Zeugnisnoten im Sommer 2007?» erfasst. Personen, die eine Berufsfachschule sowie eine Berufsmaturitätsschule besuchten, sollten die Noten der Berufsfachschule auf der gängigen sechsstufigen Notenskala (6: sehr gut, 4: genügend, 1: sehr schwach) angeben (Deutsch: $M=4.94$, $\mathrm{SD}=.46$; Mathematik bzw. Fachrechnen: $M=4.76, \mathrm{SD}=.74)$. Bei Berufslernenden wurde statt Deutsch die Note im Allgemeinbildenden Unterricht (ABU) und statt Mathematik die Note im Fachrechnen (berufskundlicher Unterricht) erfragt.

Die Arbeitssituation wurde aufgrund von neun Items erfasst. Die Hauptachsenanalyse ergab drei Faktoren mit je drei Items (Varianzaufklärung 67\%): der Faktor Neuartigkeit bestand zum Beispiel aus dem Item «Meine Arbeit ist sehr abwechslungsreich» (Alpha=.81, M=3.38, SD=.47). Der Faktor Mitsprache umfasste zum Beispiel das Item "Meine Arbeit erlaubt Mitsprache, welche Arbeit ich machen muss» (Alpha=.71, M=2.66, SD=.74). Der Faktor Belastung setzte sich unter anderem aus dem Item «meine Arbeit überfordert mich» zusammen (Alpha=.54, M=1.77, SD=.53). 
Die Zufriedenheit mit dem Berufsbildner (Neuenschwander et al., 1999) umfasste fünf Items wie zum Beispiel «Mit meinem Berufsbildner bzw. meiner Berufsbildnerin bin ich sehr zufrieden» (Alpha=.88, M=3.22, SD=.56). Die Hauptachsenanalyse ergab einen Faktor und erklärte 67\% der Varianz.

\section{Durchfuibrung}

Die Datenerhebung wurde abgestimmt auf die Erreichbarkeit der Jugendlichen je nach Messzeitpunkt unterschiedlich organisiert: Bei der ersten Datenerhebung besuchte ein Mitglied der Projektgruppe jede Klasse. Für die Datenerhebung 2006 wurden die gleichen Jugendlichen aus verschiedenen Schulklassen und Schulhäusern zu Befragungsgruppen zusammengefasst und befragt. Jugendliche, die in keiner solchen Befragungsgruppe mitmachen konnten, erhielten den Fragebogen per Post zugeschickt. Es wurde einmal gemahnt. Die Erhebung im Winter 2007 wurde im Rahmen einer Internetbefragung klassenweise oder postalisch durchgeführt. Alle Jugendlichen erhielten einen Zugangscode mit Passwort, mit dem sie sich in einen Online-Fragebogen einloggen konnten. Die Jugendlichen wurden einmal schriftlich gemahnt und erhielten bei Bedarf telefonische Unterstützung. Die Datenerhebung 2008 vollzog sich zweistufig: (1) Den Jugendlichen wurden die Fragebogen zuschickt. Ein Warengutschein von CHF 10.- lag bei. Zusätzlich wurde eine Verlosung unter den antwortenden Jugendlichen angekündigt, dessen Hauptgewinn ein Reisegutschein im Wert von CHF 600.- war. (2) Jugendliche, die nach zwei brieflichen Mahnungen nicht geantwortet hatten, wurden telefonisch von einem Mitglied der Projektgruppe konsultiert. Diese bekamen die Möglichkeit, die schriftlichen Fragen in einem gekürzten Telefoninterview zu beantworten.

\section{Auswertungsstrategie}

Die Datenauswertung erfolgte in der Reihenfolge der formulierten Hypothesen. Die Validierungshypothese wurde mit Pearson-Korrelationen überprüft. Die anderen Hypothesen wurden mit stufenweisen multiplen Regressionsanalysen (OLS) getestet. Dabei wurden die Prädiktoren im Sinne der postulierten Hypothesen blockweise in die Regressionsgleichungen einbezogen. Grundsätzlich wurde auf Strukturgleichungsmodelle verzichtet, weil die Stichprobengrösse dies nicht zuliess. Weil die missing values unsystematisch verteilt waren, wurden nur die Personen mit vollständigen Datensätzen in die Auswertung einbezogen (vgl. auch Lüdtke, Robitzsch, Trautwein \& Köller, 2007).

\section{Ergebnisse}

Zur Prüfung der Validierungshypothese wurden Korrelationsanalysen gerechnet. Die wahrgenommene Passung korrespondierte recht hoch mit der Produktivität in der Ausbildungs- bzw. Arbeitssituation ( $\mathrm{r}=.73$, $\mathrm{p}<.001$ ), aber auch mit Übergangskompetenzen $(\mathrm{r}=.43, \mathrm{p}<.001)$, subjektivem Wohlbefinden $(\mathrm{r}=.39, \mathrm{p}<.001)$ und Ausbildungszufriedenheit $(\mathrm{r}=.41, \mathrm{p}<.001)$. Die Korrelation mit der Kon- 
trollüberzeugung $(\mathrm{r}=.37, \mathrm{p}<.001)$ war ebenfalls substanziell. Interessant war auch, dass die Produktivität in der Ausbildungs- bzw. Arbeitssituation ebenfalls mit Wohlbefinden $(r=.40)$ und Ausbildungszufriedenheit $(r=.50)$ korrelierte. Wer eine hohe Passung zwischen den eigenen Fähigkeiten und Interessen und der Ausbildung bzw. dem Beruf wahrnahm, war zufriedener, motivierter und traute sich eher zu, die angefangene Ausbildung abzuschliessen (Kontrollüberzeugung). Die subjektive Passung war offenbar nicht nur ein Indikator für einen gelungenen Übergang in die Sekundarstufe II, sondern auch ein Prädiktor für den Ausbildungserfolg.

Tabelle 1: Validierung der Passungswahrnehmung (2. Lehrjahr/11. Schuljahr): Deskriptive Statistik und Pearson-Korrelationen

\begin{tabular}{|l|l|l|l|l|l|l|l|l|}
\hline $\mathrm{N}=\mathbf{1 7 6}$ & $\mathrm{M}$ & SD & 2 & 3 & 4 & 5 & 6 & 7 \\
\hline 1 Passungswahrnehmung & 3.52 & .40 & $.73^{* * *}$ & $.43^{* * *}$ & $.39^{* * *}$ & $.41^{* * *}$ & $.37^{* * *}$ & $.19^{*}$ \\
\hline 2 Produktivität & 2.28 & .39 & & $.47^{* * *}$ & $.40^{* * *}$ & $.50^{* * *}$ & $.33^{* * *}$ & $.36^{* * *}$ \\
\hline 3 Übergangskompetenzen & 3.37 & .36 & & & $.35^{* * *}$ & $.41^{* * *}$ & $.29^{* * *}$ & $.17^{*}$ \\
\hline 4 Wohlbefinden & 3.21 & .38 & & & & $.29^{* * *}$ & $.53^{* * *}$ & .11 \\
\hline 5 Ausbildungszufriedenheit & 5.77 & 1.01 & & & & & $.24^{* * *}$ & $.15^{*}$ \\
\hline 6 Kontrollüberzeugung & 3.20 & .60 & & & & & & .05 \\
\hline 7 Investitionsbereitschaft & 3.29 & .54 & & & & & & \\
\hline
\end{tabular}

Legende: ${ }^{* * *}$ : $\mathrm{p}<.001,{ }^{* *}: \mathrm{p}<.01,{ }^{*}: \mathrm{p}<.05, \uparrow: \mathrm{p}<.10$

Im nächsten Schritt rückte die Frage ins Zentrum, wie sich die Passung zwischen den Ausbildungsverläufen veränderte (Ausbildungstyphypothese). Die Stabilität der Passungswahrnehmung war eher tief, wenn auch signifikant $\left(\mathrm{r}_{\mathrm{t} 2-\mathrm{t} 3}=.26\right.$,

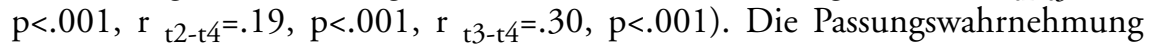
hing offenbar wesentlich vom Ausbildungskontext ab. Zur Prüfung der Ausbildungshypothese wurde eine einfaktorielle Varianzanalyse mit Messwiederholung gerechnet. Die Gruppierungsvariable bildete der Schultyp im ersten nachobligatorischen Ausbildungsjahr (entspricht dem 10. Schuljahr, Brückenangebot $\mathrm{N}=67$, Berufsbildung $\mathrm{N}=146$, Gymnasium $\mathrm{N}=47$ ). Die abhängige Variable war die Passungswahrnehmung zum zweiten und dritten Messzeitpunkt (d.h. 9. und 10. Schuljahr). Es flossen alle Jugendlichen in diese Analyse ein, die zum zweiten und dritten Messzeitpunkt an der Befragung teilgenommen hatten. Es ergab sich ein signifikanter Haupteffekt $\left(\mathrm{F}_{\mathrm{Gruppe}}=4.2, \mathrm{df}=2,257, \mathrm{p}<.06, \mathrm{R}^{2}=3.2 \%\right)$, kein Zeiteffekt und kein Interaktionseffekt (Zeit x Gruppe). Die Mittelwerte zeigten, dass die Passungswahrnehmung von Jugendlichen in Mittelschulen und Lehren höher war als diejenige von Jugendlichen in einer Übergangslösung. Offenbar vermag die qualifizierende Ausbildung in der Sekundarstufe II in höherem Ausmass die alterstypischen Bedürfnisse von Jugendlichen zu befriedigen als Brückenangebote, die von Jugendlichen eher als unangenehme Verlegenheitslö- 
sungen interpretiert werden, wobei sich dieser Effekt bereits im letzten Schuljahr der Volksschule angebahnt hat (Neuenschwander et al., 2007a).

Zur Prüfung der dritten Hypothese (Perspektive der Kompetenzentwicklung) wurde zuerst die Interkorrelationsmatrix gerechnet. Gemäss Tabelle 2 korrelierten die durchschnittlichen Zeugnisnoten aller Fächer im 5. Schuljahr mit der Passungswahrnehmung im 10. Schuljahr/1. Lehrjahr mit $r=.15(\mathrm{p}<.05)$ signifikant, aber nicht sehr stark. Die Korrelation der Gesamtnote aller Fächer zu den Fähigkeitsselbstkonzepten in Deutsch und Mathematik ist nicht signifikant. Die selbstbeurteilte Konfliktlösungsfähigkeit im 6. Schuljahr korrelierte signifikant mit der Tendenz zu aggressivem Verhalten im 9. Schuljahr negativ ( $r=-.17$, $\mathrm{p}<.05)$. Alle berücksichtigten Prädiktoren korrelierten signifikant mit der Passungswahrnehmung im 10. Schuljahr/1. Lehrjahr.

Tabelle 2: Deskriptive Statistik und Pearson-Korrelationen zwischen den postulierten Prädiktoren in der Perspektive der Kompetenzentwicklung

\begin{tabular}{|c|c|c|c|c|c|c|c|}
\hline $\mathrm{N}=206$ & M & SD & 2 & 3 & 4 & 5 & 6 \\
\hline 1 Gesamtnoten $(5 . \mathrm{Sj})$ & 5.13 & .41 & .01 & .01 & .08 & -.06 & $.15^{*}$ \\
\hline 2 Konfliktlösungsfähigkeit (6. Sj) & 3.08 & .39 & 1 & .05 & -.02 & $-.17^{*}$ & $.18^{*}$ \\
\hline 3 Fähigkeitsselbstkonzept Deutsch ${ }_{(9 . \mathrm{Sj})}$ & 3.07 & .41 & & 1 & .00 & $-.13 \dagger$ & $.27^{* * *}$ \\
\hline 4 Fähigkeitsselbstkonzept Math $\left(9 . \mathrm{Sj}_{\mathrm{j}}\right.$ & 2.82 & .55 & & & 1 & .01 & $.22^{* * *}$ \\
\hline 5 Aggressives Verhalten ${ }_{(9 . \mathrm{Sj})}$ & 1.76 & .73 & & & & 1 & $-.22^{* *}$ \\
\hline 6 Passungsw. ${ }_{(10 . \mathrm{Sj})}$ & 3.47 & .50 & & & & & 1 \\
\hline
\end{tabular}

Legende: ${ }^{* * *}$ : p<.001, ${ }^{* *}: \mathrm{p}<.01,{ }^{*}: \mathrm{p}<.05, \uparrow: \mathrm{p}<.10$, Sj: Schuljahr, Passungsw.: Passungswahrnehmung

Danach wurden schrittweise multiple OLS-Regressionsanalysen gerechnet. Gemäss Tabelle 3 konnte die Passungswahrnehmung im 11. Schuljahr/2. Lehrjahr durch die Noten und die Konfliktlösungsfähigkeit im 6. Schuljahr signifikant vorhergesagt werden, die Varianzaufklärung betrug nur 5\%. Im zweiten Schritt wurden zusätzlich die Fähigkeitsselbstkonzepte in Deutsch und Mathematik sowie der Faktor aggressives Verhalten im 9. Schuljahr in die Gleichung einbezogen. Der Effekt der Noten verschwand nun, die drei neuen Prädiktoren wurden signifikant. Das Modell erklärte 16\%. Die Fähigkeitsselbstkonzepte und das aggressive Verhalten unterdrückten den Effekt der Noten. Allerdings blieb der Faktor Konfliktlösungsfähigkeit signifikant. Im dritten Schritt wurde die Passungswahrnehmung im 10. Schuljahr zusätzlich in der Gleichung berücksichtigt und wurde signifikant (beta=.33, p<.001). Die Prädiktoren dienten in dieser Analyse nicht mehr der Erklärung des Niveaus der Passungswahrnehmung, sondern der Veränderung der Passungswahrnehmung. Von den früheren Variablen war nur noch der Faktor aggressives Verhalten signifikant (beta=-.15, p<.05), wobei dessen Wert deutlich abgenommen hatte. Fähigkeitsselbstkonzepte und aggressives Verhalten vermochten also die Passungswahrnehmung vorherzusagen, aber nur 
das aggressive Verhalten erklärte die Veränderung der Passungswahrnehmung. Im vierten Schritt wurden zusätzlich Nationalität und Geschlecht als Kontrollvariablen in die Gleichung einbezogen, ohne aber das frühere Ergebnis zu verändern.

Tabelle 3: Determinanten zur Entstehung der Passungswahrnehmung im 11. Schuljahr/2. Lehrjahr in der Perspektive der Kompetenzentwicklung (standardisierte Regressionskoeffizienten)

\begin{tabular}{|c|c|c|c|c|}
\hline $\mathrm{N}=190$ & 1. Schritt & 2. Schritt & 3. Schritt & 4. Schritt \\
\hline Gesamtnoten $_{\left(5 . S_{j}\right)}$ & $.14^{*}$ & .11 & .07 & .07 \\
\hline Konfliktlösungsfähigkeit (6. Sj) & $.15^{*}$ & $.12^{*}$ & .08 & .08 \\
\hline Fähigkeitsselbstkonzept Deutsch ${ }_{\left(9 . \mathrm{Sj}_{\mathrm{j}}\right.}$ & - & $.20^{* *}$ & $.12 \dagger$ & $.12 \dagger$ \\
\hline \begin{tabular}{|l} 
Fähigkeitsselbstkonzept Math ${ }_{(9 .}$ Sj) \\
\end{tabular} & - & $.18^{* *}$ & .11 & .11 \\
\hline Aggressives Verhalten (9. Sj) & - & $-.20^{* *}$ & $-.15^{*}$ & $-.16^{*}$ \\
\hline Passungsw. (10. Sj) & & & $.33^{* * *}$ & $.33^{* * *}$ \\
\hline Geschlecht $_{\left(9 . \mathrm{Sj}_{\mathrm{j}}\right.}$ (1: männlich, 2: weiblich) & - & - & - & -.03 \\
\hline Staatsangehörigkeit $_{(9 . \mathrm{Sj})}(0: \mathrm{CH}, 1$ : Ausland $)$ & - & - & - & .02 \\
\hline$F, d f, p$ & $\begin{array}{c}4.4^{* *}, 2 \\
187\end{array}$ & $\begin{array}{c}6.9^{* * *}, 5 \\
184\end{array}$ & $\begin{array}{c}10.2^{* * *}, 6 \\
183\end{array}$ & $\begin{array}{c}7.6^{* * *}, 8 \\
181\end{array}$ \\
\hline $\mathrm{R}^{2}$ korr & $5 \%$ & $16 \%$ & $25 \%$ & $25 \%$ \\
\hline
\end{tabular}

Legende: *** ${ }^{* *}<.001,{ }^{* *}: \mathrm{p}<.01,{ }^{*}: \mathrm{p}<.05, \dagger: \mathrm{p}<.10$, Sj: Schuljahr

Zur Prüfung der vierten Hypothese (Perspektive des Arbeitsplatzes) wurden zuerst Korrelationen zwischen den Prädiktoren gerechnet (Tabelle 4). Interessanterweise korreliert das Prestige der Berufslehre mit der Zufriedenheit mit dem Berufsbildner $(\mathrm{r}=.18, \mathrm{p}<.05)$. Dann wurden wiederum schrittweise multiple OLS-Regressionsanalysen gerechnet. Im ersten Schritt wurde gemäss Tabelle 5 die Passungswahrnehmung im 1. Lehrjahr zur Erklärung der Passungswahrnehmung im 2. Lehrjahr verwendet (Varianzaufklärung knapp 8\%). Im zweiten Schritt wurden die letzten Zeugnisnoten in Deutsch/ABU und Mathematik/ Fachrechnen sowie die Konfliktlösefähigkeit berücksichtigt. Die Varianzaufklärung nahm zu (12\%). Im dritten Schritt wurde das Prestige der Berufslehre, welche die Lernenden absolvierten, zusätzlich einbezogen. Es erklärte die Passungswahrnehmung aber nicht. Gleichzeitig wurden die Arbeitsplatzmerkmale Neuartigkeit, Mitsprache und Belastung sowie die Zufriedenheit mit dem Berufsbildner in die Gleichung aufgenommen. Die Varianzaufklärung stieg nun deutlich (40\%). Im letzten Schritt wurden zu Kontrollzwecken das Geschlecht und die Staatsangehörigkeit einbezogen. Die Staatsangehörigkeit wurde signifikant. Bei den ausländischen Berufslernenden nimmt die Passungswahrnehmung im Vergleich zu den Einheimischen vom ersten zum zweiten Lehrjahr eher ab. Es wurde kein Geschlechtseffekt gefunden. Die Koeffizienten zum Arbeitsplatz ver- 
änderten sich unwesentlich. Die Passungswahrnehmung im vorangegangenen Lehrjahr war aber nicht signifikant. Das letzte Modell erklärte 43\% der Veränderung der Passungswahrnehmung vom 1. zum 2. Lehrjahr, was als erklärungsstarkes Modell bewertet werden kann. Die Arbeitsplatzmerkmale waren dafür hauptverantwortlich. Der Effekt der Noten blieb auch nach Einbezug der Arbeitsplatzmerkmale tendenziell $(\mathrm{p}<.10)$ erhalten, während der Effekt der Konfliktlösefähigkeit nach Einbezug der Arbeitsplatzmerkmale unterdrückt wurde.

Tabelle 4: Deskriptive Statistik und Pearson-Korrelationen zwischen den Prädiktoren im 1. bzw. 2. Lehrjahr in der Perspektive des Arbeitsplatzes

\begin{tabular}{|c|c|c|c|c|c|c|c|c|c|c|c|}
\hline $\mathrm{N}=126$ & $\mathrm{M}$ & SD & 2 & 3 & 4 & 5 & 6 & 7 & 8 & 9 & 10 \\
\hline 1. Passungsw. (1. Lj) & 3.49 & .48 & $.19^{*}$ & -.06 & $.15 \dagger$ & .08 & -.05 & $-.13 \dagger$ & $.19^{*}$ & $-.21^{*}$ & $-.15 \dagger$ \\
\hline $\begin{array}{l}\text { 2. Zeugnisnoten (Deutsch } \\
\text { / ABU und Math/Fach- } \\
\text { rechnen) (1. Lj) }\end{array}$ & 5.00 & 4.48 & 1 & .03 & -.06 & .05 & .01 & -.07 & .06 & .08 & -.07 \\
\hline $\begin{array}{l}\text { 3. Konfliktlösungsfähig- } \\
\text { keit (2. Lj) }\end{array}$ & 3.07 & .39 & & 1 & -.03 & .06 & .08 & $-.16 \dagger$ & $.15 \dagger$ & $.15 \dagger$ & -.12 \\
\hline $\begin{array}{l}\text { 4. Prestige Berufslehre } \\
\text { (SIOPS) }_{(2 . \mathrm{Lj})}\end{array}$ & $\begin{array}{c}40.2 \\
0\end{array}$ & 6.97 & & & 1 & .03 & -.08 & -.01 & $.18^{*}$ & -.04 & -.07 \\
\hline $\begin{array}{l}\text { 5. Arbeitsplatz: } \\
\text { Neuartigkeit }(2 . \mathrm{Lj})\end{array}$ & 3.43 & .44 & & & & 1 & $.38^{* *}$ & -.06 & $.48^{* * *}$ & -.02 & -.12 \\
\hline $\begin{array}{l}\text { 6. Arbeitsplatz: } \\
\text { Mit-sprache }\left(2 . \mathrm{L}_{\mathrm{j}}\right)\end{array}$ & 2.79 & .62 & & & & & 1 & $-.19^{*}$ & $.31^{* * *}$ & -.01 & -.03 \\
\hline $\begin{array}{l}\text { 7. Arbeitsplatz: } \\
\text { Belastung (2. Lj) }\end{array}$ & 1,99 & .51 & & & & & & 1 & -.02 & -.02 & .03 \\
\hline $\begin{array}{l}\text { 8. Zufriedenheit } \\
\text { Berufsbildner/-in (2. Lj) }\end{array}$ & 3.28 & .48 & & & & & & & 1 & -.07 & -.14 \\
\hline $\begin{array}{l}\text { 9. Geschlecht (2. Lj) } \\
\text { (1: männlich, 2: weiblich) }\end{array}$ & 1.51 & .50 & & & & & & & & 1 & $-.20^{*}$ \\
\hline $\begin{array}{l}\text { 10. Staatsangehörigkeit (2. Lj) } \\
\text { (1: CH, 2: Ausland) }\end{array}$ & 1.06 & .24 & & & & & & & & & 1 \\
\hline
\end{tabular}

Legende: ${ }^{* * *}: \mathrm{p}<.001,{ }^{* *}: \mathrm{p}<.01,{ }^{*}: \mathrm{p}<.05, \dagger: \mathrm{p}<.10, \mathrm{ABU}$ : allgemeinbildender Unterricht, 1. Lj: 1. Lehrjahr

\section{Diskussion}

Der vorliegende Beitrag geht den Fragen nach, wie Lernende in der Sekundarstufe II eine Passung zu ihrer Ausbildung herstellen. Die Passung zwischen Lernenden und ihrem Beruf ist nicht nur ein Kriterium eines erfolgreichen Berufswahlprozesses und Übergangs in die Berufsbildung (Holland, 1973), sondern bildet auch einen Indikator für Ausbildungsqualität (Eccles, 2004). Aus den Ergebnissen folgt, dass die wahrgenommene Passung ein guter Indikator ist, wie Jugendliche ihren Ausbildungsverlauf erleben und bewerten. Längsschnittliche Analysen in der Perspektive der Kompetenzentwicklung bestätigten, dass schuli- 
sche Noten und soziale Kompetenzen am Ende der Primarschule einen erfolgreichen Übergang in die nachobligatorische Ausbildung vorbereiten. In der Berufslehre bestimmen aber Arbeitsplatzmerkmale deutlich stärker die Veränderung der Passungswahrnehmung als schulische Noten und soziale Kompetenzen (Perspektive des Arbeitsplatzes).

Tabelle 5: Determinanten zur Entstehung der Passungswahrnehmung im 2. Lehrjahr in der Perspektive des Arbeitsplatzes (standardisierte Regressionskoeffizienten)

\begin{tabular}{|l|c|c|c|c|}
\hline $\mathrm{N}=126$ & 1. Schritt & 2. Schritt & 3. Schritt & 4. Schritt \\
\hline Passungsw (1. Lj) & $.29^{* * *}$ & $.27^{* *}$ & $.17^{*}$ & $.14 \dagger$ \\
\hline $\begin{array}{l}\text { Zeugnisnoten (Deutsch / ABU } \\
\text { und Math / Fachrechnen) (1. Lj) }\end{array}$ & - & $.16 \dagger$ & $.14 \dagger$ & $.13 \dagger$ \\
\hline Konfliktlösungsfähigkeit & - & $.19^{*}$ & .11 & .08 \\
\hline Prestige Berufslehre (SIOPS) & - & - & .04 & .03 \\
\hline Arbeitsplatz: Neuartigkeit & - & - & $.44^{* * *}$ & $.43^{* * *}$ \\
\hline Arbeitsplatz: Mitsprache & - & - & -.08 & -.08 \\
\hline Arbeitsplatz: Belastung & - & - & $-.23^{* *}$ & $-.23^{* *}$ \\
\hline Zufriedenheit Berufsbildner/-in & - & - & $.15 \dagger$ & .14 \\
\hline Geschlecht (1: männlich, 2: weiblich) & - & - & - & .01 \\
\hline Staatsangehörigkeit & & & & \\
\hline (1: CH, 2: Ausland) & - & - & - & $-.20^{* *}$ \\
\hline F, p & $11.4^{* * *}$ & $6.9^{* * *}$ & $11.6^{* * *}$ & $10.6^{* * *}$ \\
\hline df & 1,124 & 3,122 & 8,117 & 10,115 \\
\hline R2 korr & $8 \%$ & $12^{*} \%$ & $40 \%$ & $43 \%$ \\
\hline
\end{tabular}

Legende: ${ }^{* * *}$ : $\mathrm{p}<.001,{ }^{* *}: \mathrm{p}<.01,{ }^{*}: \mathrm{p}<.05, \dagger: \mathrm{p}<.10$

Obwohl die Mensch-Umwelt-Passung immer wieder als Erfolgskriterium von Entwicklungsverläufen und Übergangs- und Selektionsprozessen vorgeschlagen worden ist (Eccles, 2004), wurde sie bisher im Schweizer Berufsbildungskontext nicht untersucht. Passung wurde als Korrespondenz von Ausbildungskontext und individuellen Kompetenzen und Interessen konzipiert. Wie die Intelligenzforschung postuliert, bilden Kompetenzen wesentliche Grundlagen dafür, dass sich Jugendliche an Umweltanforderungen anpassen können (Sternberg, 1984). Es wurde daher ein neues Erklärungsmodell entwickelt und längsschnittlich gezeigt, dass schulische Noten und soziale Kompetenzen die Passung in der Sekundarstufe II vorbereiten. Überdies belegen die Ergebnisse die relative Unabhängigkeit der schulischen und sozialen Kompetenzen, die auf unterschiedliche 
Art die Integration in die nachobligatorische Ausbildung begünstigen. Ein erfolgreicher Übergang in die Sekundarstufe II erfordert sowohl kognitive als auch soziale Kompetenzen, um einerseits die schulischen Leistungsanforderungen zu erfüllen, aber auch gute Beziehungen zu Berufsbildner/-in, Mitarbeitenden und anderen Lernenden in Betrieb und Schule zu pflegen. Es wird vermutet und von nicht dargestellten eigenen Ergebnissen bestätigt, dass die Passungswahrnehmung durch Kompetenzen von Jugendlichen, aber nicht durch ihre Motivationslage im Berufswahlprozess vorgesagt werden kann.

Konkret bilden gute schulische Leistungen eine Grundlage für eine hohe Passungswahrnehmung, wobei das Fähigkeitsselbstkonzept den Effekt der Noten unterdrückt. Primär das Fähigkeitsselbstkonzept ist für die Passungswahrnehmung nach dem Übergang bedeutsam (Super, 1992). Gute Konfliktlösefähigkeiten - teilweise vermittelt über geringe aggressive Verhaltenstendenzen gegenüber Gleichaltrigen - schaffen soziale Bedingungen für eine hohe Passungswahrnehmung. Die Ergebnisse zeigen, dass hohe aggressive Tendenzen nicht nur die Stärke der Passungswahrnehmung, sondern auch die Veränderung der Passungswahrnehmung vom ersten zum zweiten Lehrjahr beeinträchtigen, weil sie die Beziehungen im Betrieb und das Ausbildungsklima verschlechtern.

Während sich die Analysen zur Kompetenzentwicklung auf die Situation in der Sekundarstufe II bezogen, weil sich die Jugendlichen nach dem sechsten Schuljahr in sehr unterschiedliche Schulformen der Sekundarstufe I und II bewegten, mussten die Analysen in der Arbeitsplatzperspektive auf die Berufsbildung eingeschränkt werden. Denn Mittelschulen und Lehrbetriebe sind durch sehr unterschiedliche Arbeitsplatzmerkmale charakterisiert. Die Ergebnisse zeigen, dass die Effekte der Konfliktlösekompetenz auf die Veränderung der Passungswahrnehmung durch Merkmale des Arbeitsplatzes im Lehrbetrieb vollständig kompensiert werden. Die sozialen Kompetenzen von Jugendlichen tragen zu einer günstigen Arbeitsplatzgestaltung bei, welche ihrerseits die Passungswahrnehmung beeinflussen. Diese Suppressionseffekte zeigen, dass soziale Kompetenzen und Arbeitsplatzmerkmale miteinander interagieren und die Passungswahrnehmung beeinflussen.

Zentral sind Arbeitsplatzmerkmale wie Neuartigkeit und Abwechslung der betrieblichen Arbeit sowie geringe Belastungen. Die Beziehung zum Berufsbildner/zur Berufsbildnerin ist nur tendenziell signifikant. Diese korrelierte bivariat mit der Passungswahrnehmung, aber ihr Effekt wird durch die Konfliktlösekompetenz und die frühere Passung unterdrückt. Das Prestige der Ausbildung ist hingegen für die Passungswahrnehmung unerheblich. Die Jugendlichen erleben eine geringere Passung in einer nicht zertifizierenden Sekundarstufe II-Ausbildung (Brückenangebot).

Zusammenfassend zeigen die Ergebnisse, dass gute schulische Noten und Konfliktlösekompetenzen am Ende der Primarschule Kindern erlauben, ein günstiges Ausbildungsumfeld auszuwählen bzw. herzustellen (Brandtstädter, 1999), in welchem sie eine hohe Passung wahrnehmen können. Damit wird eine 
Voraussetzung für eine hohe Ausbildungszufriedenheit und einen erfolgreichen Ausbildungsabschluss geschaffen. Die Analysen in der Perspektive des Arbeitsplatzes erreichten aber deutlich höhere Varianzaufklärungen als diejenigen in der Perspektive der Kompetenzentwicklung, die sich auf einen Zeitraum von mehreren Jahren bezogen. Weil die Konzepte in der Arbeitsplatzperspektive gleichzeitig gemessen worden sind, konnte die postulierte Kausalitätsrichtung nicht überprüft werden. Die Konzepte in der Arbeitsplatzperspektive erklärten aber querschnittlich die Passungswahrnehmung deutlich stärker als die Noten und die Konfliktlösekompetenzen.

Einschränkend muss angemerkt werden, dass die Analysen auf selbstberichteten Daten basieren. Damit rückt das Erleben der untersuchten Jugendlichen ins Zentrum. Es können aber keine Aussagen über die objektiven Gegebenheiten gemacht werden. Leider konnte der Zusammenhang zwischen schulischen Kontextmerkmalen und der Passungswahrnehmung in Mittelschulen aus Platzgründen nicht analysiert werden. Folgeuntersuchungen sollten dieser Frage nachgehen. Ebenfalls wären Analysen zur Passungswahrnehmung nach Abschluss der Sekundarstufe II wichtig (vgl. dazu Gerber-Schenk, Rottermann \& Neuenschwander, 2010). Damit können Bezüge zu traditionellen Berufswahltheorien, auch zu Selektionsprozessen im Lehrstellen- und Arbeitsmarkt hergestellt werden. Die Weiterführung der Passungsforschung im Kontext der Schweizer Berufsbildung erscheint aufgrund der Ergebnisse vielversprechend.

\section{Anmerkungen}

1 Ich danke dem Schweizerischen Nationalfonds (Projektnummern 10013-107733 sowie 100014-116026) und der Pädagogischen Hochschule Bern (Projektnummer 0101s017) für die finanziellen Beiträge an die Untersuchung.

2 ISEI: Standard International Socio-Economic Index of Occupational Status

\section{Literatur}

Alsaker, F. (2003). Quälgeister und ihre Opfer. Bern: Huber.

Brandtstädter, J. (1999). The self in action and development: Cultural, biosocial, and ontogenetic bases of intentional self-development. In J. Brandtstädter \& R. M. Lerner (Ed.), Action and self-development: Theory and research through the life span (pp. 37-66). Thousand: Oaks.

Buff, A. (1991). Persönlichkeitsentwicklung im Umfeld des Übertritts in die Sekundarstufe I. Zürich: ADAG.

Busshoff, L. (1998). Berufsberatung als Unterstützung von Übergängen in der beruflichen Entwicklung. In R. Zihlmann (Hrsg.), Berufswahl in Theorie und Praxis (S. 9-86). Zürich: SABE.

Eccles, J. S. (2004). Schools, academic motivation, and stage-environment fit. In R. M. Lerner \& L. Steinberg (Ed.), Handbook of adolescent psychology (pp. 125-153). Hoboken: John Wiley \& Sons.

Eccles, J. S., Midgley, C., Wigfield, A., Buchanan, C. M., Reuman, D., Flanagan, C. \& Mac Iver, D. (1993). Development during adolescence: The impact of stage-environment fit on young adolescents' experiences in schools and in families. American Psychologist, 48 (2), 90-101.

Gerber-Schenk, M., Rottermann, B. \& Neuenschwander, M. P. (2010). Passungswahrnehmung, Selbstkonzept und Jugendarbeitslosigkeit. In M. P. Neuenschwander \& H.-U. Grunder (Hrsg.), Schulübergang und Selektion (S. 121-130). Chur: Rüegger. 
Gottfredson, L. S. (1996). Gottfredsons's theory of circumscription and compromise. In D. Brown \& L. Brooks (Ed.), Career choice and development (pp. 179-232). San Francisco: Jossey-Bass Inc.

Grob, A., Lüthi, R., Kaiser, F. G., Flammer, A., Mackinnon, A. \& Wearing, A. (1991). Berner Fragebogen zum Wohlbefinden Jugendlicher (BFW). Diagnostica, 37, 66-75.

Heinz, W. R. (Hrsg.). (2000). Übergänge (3. Beiheft zur Zeitschrift für Soziologie der Erziehung und Sozialisation). Weinheim: Juventa.

Helmke, A. \& Weinert, F. (1997). Bedingungsfaktoren schulischer Leistungen. In F. E. Weinert (Hrsg.), Enzyklopädie der Psychologie (Bd. 3, Psychologie des Unterrichts und der Schule, S. 71-176). Göttingen: Hogrefe.

Herzog, W., Neuenschwander, M. P. \& Wannack, E. (2006). Berufswahlprozess. Wie sich Jugendliche auf ihren Beruf vorbereiten. Bern: Haupt.

Holland, J. L. (1973). Making vocational choices. Englewood Cliffs: Prentice-Hall.

Kristof-Brown, A. L., Zimmerman, R. D. \& Johnson, E. C. (2005). Consequences of individual's fit at work: A meta-analysis of person-job, person-organization, person-group, and person-supervisor fit. Personnel Psychology, 58 (2), 1-342.

Labudde, P., Herzog, W., Neuenschwander, M. P., Violi, E. \& Gerber, C. (2000). Girls and physics: Teaching and learning strategies tested by classroom intervention in grade 11 . International Journal of Science Education, 22 (2), 143-157.

Lüdtke, O., Robitzsch, A., Trautwein, U. \& Köller, O. (2007). Umgang mit fehlenden Werten in der psychologischen Forschung. Probleme und Lösungen. Psychologische Rundschau, 58 (2), 103-117.

Neuenschwander, M. P. (2005). Unterrichtssystem und Unterrichtsqualität. Bern: Haupt.

Neuenschwander, M. P. \& Frank, N. (2009). Familie-Schule-Beruf(FASE B)-Dokumentation der Schülerbefragung 2008 (Forschungsbericht). Solothurn: Pädagogische Hochschule Nordwestschweiz.

Neuenschwander, M. P. \& Malti, T. (2009). Selektionsprozesse beim Übergang in die Sekundarstufe I und II. Zeitschrift für Erziehungswissenschaft, 12 (2), 216-232.

Neuenschwander, M. P. \& Wismer, N. (2010). Selektionskriterien: Wichtige Rolle der überfachlichen Kompetenzen. Panorama, 101, 16-17.

Neuenschwander, M. P., Angehrn, F., Bugnard, I., Gehrig, M., Heinss, R., Frey, M. \& Gasser, L. (2007a). Familiäre Bedingungen von Schülerleistungen. Universität Zürich, Jacobs center for productive youth development.

Neuenschwander, M. P., Goltz, S., Balmer, T., Gasser, A., Hirt, U., Ryser, H., Wartenweiler, H. (2003). Dokumentation der Schülerbefragung (Forschungsbericht). LLB Bern.

Neuenschwander, M. P., Schaub, S. \& Angehrn, F. (2007b). Familiäre Bedingungen von Schülerleistungen. Dokumentation des Schülerfragebogens (Forschungsbericht). Universität Zürich, Jacobs center for productive youth development.

Neuenschwander, M. P., unter Mitarbeit von Holder, M., Engelage, S., Guggisberg, J., KunzMakarova, E. et al. (1999). Schule und Identität im Jugendalter II (Forschungsbericht Nr. 20). Universität Bern, Institut für Pädagogik.

Piaget, J. (1947). La psychologie de l'intelligence. Paris: Colin.

Pinquart, M., Juang, L. P. \& Silbereisen, R. K. (2003). Self-efficacy and successful school to work transition: A longitudinal study. Journal of Vocational Behavior, 63 (3), 329-346.

Sternberg, R. J. A. (1984). Toward a triarchic theory of human intelligence. Behavioral and Brain Sciences, 7, 269-287.

Super, D. (1992). Toward a comprehensive theory of career development. In D. H. Montross \& C. J. Shinkman (Ed.), Career development: Theory and practice (pp. 35-64). Springfield: Thomas.

Treiman, D. J. (1977). Occupational prestige in comparative perspective. New York: Academic Press.

Schlagworte: Transition, Passung, Berufslehre, Sozialkompetenz, Längsschnittstudie, Sekundarstufe 


\title{
Les déterminants du sentiment d'être adéquat à son environnement après la transition au secondaire II
}

\begin{abstract}
Résumé
La perception qu'ont les jeunes d'être en adéquation avec leur univers de formation est non seulement un critère d'une transition réussie dans la formation professionnelle, mais également de satisfaction au travail et de productivité dans l'entreprise. Sur la base du projet de recherche "famille-école-profession" (en allemand FASE B), nous examinons dans quelle mesure la perception d'adéquation est prédite par (1) par les compétences scolaires et sociales antérieures (perspective développementale) et (2) par des facteurs relatifs à la place de travail (perspective contextuelle). L'analyse de régression pas à pas des données longitudinales montre que des caractéristiques contextuelles de l'entreprise expliquent plus de variance du sentiment d'être adéquat que l'approche développementale des compétences. Les résultats suggèrent que des compétences élevées en résolution de conflits contribuent à un meilleur aménagement de la place de travail qui, en retour, améliore la perception d'être adéquat.
\end{abstract}

Mots clés: Transition, perception d'adéquation, compétence sociale, étude longitudinale, enseignement secondaire, formation professionnelle

\section{Determinanti della percezione di adeguatezza nella transizione al Secondario II}

\section{Riassunto}

Un'alta percezione di adeguatezza (fit) tra il livello di sviluppo del giovane e il suo contesto formativo non è solo in requisito per una buona transizione alla formazione professionale, ma anche per raggiungere la soddisfazione nel proprio lavoro e una buona produttività. Sulle basi del progetto Famiglia-Scuola-Lavoro (FASE B) si cerca di (1) prevedere il livello di percezione di adeguatezza a partire dalle competenze accademiche e sociali, nell'ottica dello sviluppo di competenze; (2) determinare la percezione di adeguatezza tramite variabili legate all'impresa, considerando il posto di lavoro. L'analisi di regressione dei dati longitudinali mostra che la varianza nella percezione di adeguatezza viene spiegata in più larga misura da condizioni legate al posto di lavoro che allo sviluppo delle competenze. I risultati indicano che una buona competenza di soluzione dei conflitti da parte dei giovani contribuisce a generare un buon clima di lavoro, che a sua volta ha un influsso positivo sulla percezione di adeguatezza.

Parole chiave: Transizione, adeguatezza, Professione docente, Competenze sociali, studio longitudinale, secondario 


\section{Determinants of Perceived Fit after Transition to Upper Secondary Education}

\section{Abstract}

A high perceived stage-environment-fit does not only indicate a successful transition to vocational education and training (VET), but also predicts job satisfaction and productivity in a company. Based on the "family-school-job» research (FASE B in German), we study to what extend the perceived fit is predicted by (1) academic and social competences in primary school (competence development approach), and (2) by managerial determinants (workplace context approach). Results of stepwise regression analyses show that more perceived fit variance can be explained with the workplace approach than with the developmental approach of competence. They also indicate that students' conflict resolution skills contribute to the workplace climate that in turn influences the development of the perceived fit.

Key words: Transition, stage-environment-fit, social competence, longitudinal study, secondary education, vocational education and training 